\title{
Functionality of the spindle checkpoint during the first meiotic division of mammalian oocytes
}

\author{
S. Brunet ${ }^{1 *}$, G. Pahlavan ${ }^{1}$, S. Taylor ${ }^{2}$ and B. Maro ${ }^{1 \dagger}$ \\ ${ }^{1}$ Laboratoire de Biologie Cellulaire du Developpement, UMR 76229, Quai Saint-Bernard, Bat. C, 5eme \\ etage, Case 24, 75252 Paris Cedex 05, France; and ${ }^{2}$ University of Manchester, School of Biological \\ Sciences, 2205 Stopford Building, Oxford Road, Manchester M13 9PT, UK
}

The spindle checkpoint ensures accurate chromosome segregation by delaying anaphase until all chromosomes are correctly aligned on the microtubule spindle. Although this mechanism is conserved throughout eukaryotic evolution, it is unclear whether it operates during meiosis in female mammals. The results of the present study show that in mouse oocytes spindle alterations prevent both chromosome segregation and MPF (M phase promoting factor) inactivation during the first meiotic $M$ phase. Moreover, the spindle checkpoint component budding uninhibited by benzimidazole 1 (BUB1) localizes to kinetochores and is phosphorylated until anaphase of both meiotic $\mathrm{M}$ phases. Both localization and phosphorylation are similar to those observed in oocytes at microtubule depolymerization. In addition, the kinetochore localization and phosphorylation of BUB1 do not depend on the MOS/.../MAPK pathway. These data indicate that the spindle checkpoint is probably active during meiotic maturation in mouse oocytes. BUB1 remains associated with kinetochores and is phosphorylated during the metaphase arrest of the second meiotic $\mathrm{M}$ phase, indicating that this protein may also play a role in the natural metaphase II arrest in mammalian oocytes.

\section{Introduction}

During meiosis, two rounds of chromosome segregation occur without DNA replication, thereby producing haploid gametes. The first meiotic division is reductional and involves the segregation of homologous chromosomes while sister chromatids remain attached. The second meiotic division is equational and leads to the segregation of sister chromatids, as also occurs during mitosis. Segregation errors during either meiotic division can result in aneuploid embryos after fertilization, which in turn can have marked consequences. Specifically, in mammals, the loss or gain of an autosome can result in severe developmental, physiological and mental disturbances (Abruzzo and Hassold, 1995).

In mitosis, a quality control mechanism called the spindle assembly checkpoint ensures accurate chromosome segregation by delaying the onset of anaphase until all the chromosomes are correctly attached to the spindle through their kinetochores. The spindle checkpoint prevents anaphase by inhibiting the anaphase promoting complex/cyclosome (APC/C), an E3 ubiquitin

* Present address: Laboratoire de Biochimie Cellulaire CNRS UMR 7098, Bat.C 5eme étage, Case 265, 9, quai St Bernard, F-75 252 Paris Cedex 05, France

${ }^{\dagger}$ Correspondence

Email:maro@ccr.jussieu.fr ligase, the activity of which is required for onset of anaphase and exit from mitosis (Cleveland et al., 2003). The spindle checkpoint components budding uninhibited by benzimidazole (BUB; Hoyt et al., 1991) and mitotic arrest-deficient (MAD; Li and Murray, 1991) are conserved throughout eukaryotic evolution (Amon, 1999). In vertebrates, BUB1, BUB3, MAD1, MAD2 and BUBR1 localize transiently to kinetochores during mitosis, which is consistent with playing a role in monitoring kinetochore-microtubule interactions. MAD2 appears to respond directly to microtubule anchoring in the kinetochore plate (Waters et al., 1999), whereas BUB1 appears to be sensitive to tension across the centromere (Skoufias et al., 2001). Significantly, the amounts of BUB and MAD proteins present at kinetochores decrease after chromosome alignment. Whether dissociation of these proteins is required for checkpoint inactivation remains to be determined. What is clear is that BUB1 is phosphorylated when the spindle checkpoint is turned on (Taylor and McKeon, 1997) and that its phosphorylation is required for the formation of the MAD1-BUB1-BUB3 complex that is crucial for spindle checkpoint function (Brady and Hardwick, 2000).

Whether the spindle checkpoint operates in mammalian meiosis remains unclear. In humans, an estimated $20 \%$ of conceptuses have an abnormal chromosomal content as a consequence of errors that occur during 
female meiosis I (Angell et al., 1994; Hunt and LeMaireAdkins, 1998). In humans, as well as in mice, in which maternal age model systems have been developed, the frequency of segregation errors is strongly correlated to maternal age (Eichenlaub-Ritter et al., 1988). Subsequently, it has become generally accepted, but not formally demonstrated, that meiotic divisions in female mammals are error prone because they lack a functional spindle checkpoint.

In the present study, it is demonstrated that mouse oocytes do undergo cell cycle arrest after different types of spindle damage. In addition, it is shown that BUB1 localizes to kinetochores and is in a phosphorylated state before anaphase of both meiotic $M$ phases. In contrast to observations in Xenopus oocytes (Schwab et al., 2001), the phosphorylation and kinetochore localization of BUB1 in mouse oocytes do not require the MOS/.../MAPK pathway. These observations are all consistent with the notion that the spindle checkpoint is operational during meiotic maturation in female mammals. Finally, BUB1 remains associated with kinetochores and is phosphorylated during the metaphase II arrest, indicating that it may play a role in this natural arrest of mammalian oocytes.

\section{Materials and Methods}

\section{Oocyte culture and collection}

Oocytes blocked at prophase of the first meiotic division (germinal vesicle stage). The ovaries were removed from 8-12-week-old Swiss (Ifa Credo, St Germain sur L'Arbresle) or mos -/- (Colledge et al., 1994) female mice and transferred to prewarmed $\left(37^{\circ} \mathrm{C}\right) \mathrm{M} 2$ medium

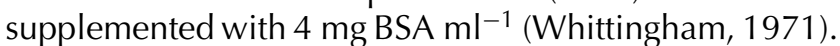
The ovarian follicles were punctured to release the enclosed oocytes, and immature oocytes displaying a germinal vesicle $(\mathrm{GV})$ were collected and cultured in $\mathrm{M} 2$ medium under liquid paraffin oil at $37^{\circ} \mathrm{C}$. Oocytes were scored for germinal vesicle breakdown (GVBD) after $1 \mathrm{~h}$ of culture and collected at different time points.

Oocytes blocked at metaphase of the second meiotic division. Metaphase II-arrested oocytes were recovered from mice superovulated by i.p. injections of equine chorionic gonadotrophin (eCG; Intervet, AN Boxmeer) and human chorionic gonadotrophin (hCG; Intervet) $48 \mathrm{~h}$ apart. Ovulated oocytes were released from the ampullae of oviducts at 14-16 h after hCG administration and the cumulus cells were dispersed by exposure to $0.1 \mathrm{~mol}$ hyaluronidase $\mathrm{I}^{-1}$ (Sigma Chemical Co., St Louis, MO). After washing, the oocytes were cultured in $\mathrm{M} 2$ medium under liquid paraffin oil at $37^{\circ} \mathrm{C}$.

Parthenogenetically activated oocytes. Oocytes were activated by exposure for $6.5 \mathrm{~min}$ to a freshly prepared
$8 \%$ ethanol solution in M2 medium (Cuthbertson, 1983). The oocytes were washed in M2 medium and cultured in $\mathrm{M} 2$ medium under liquid paraffin oil at $37^{\circ} \mathrm{C}$.

\section{Oocyte drug treatment}

Oocytes were washed and cultured in M2 medium containing Nocodazole (Sigma Chemical Co.) at either $20 \mathrm{~mol} \mathrm{I}^{-1}$ or $400 \mathrm{nmol} \mathrm{I}^{-1}$, or, alkrnatively Paclitaxel (Molecular Probes, Eugene, OR) at $20 \mathrm{nmol} \mathrm{I}^{-1}$. Oocytes were cultured in M2 medium containing Puromycin (Sigma Chemical Co.) at $20 \mu \mathrm{mol} \mathrm{I}^{-1}$, with or without $20 \mu \mathrm{mol}$ Nocodazole $\mathrm{I}^{-1}$. The drugs were removed by intensive washing of the treated oocytes in M2 medium. All experiments were performed at least twice.

\section{Immunocytochemistry}

The fixation and labelling of oocytes were performed as described by Maro et al. (1984). Oocytes were fixed and stained according to Taylor and McKeon (1997). Confocal microscopy was performed with a Leica DMR/TCS-4D instrument.

\section{Immunoblotting}

Groups of 50 oocytes were washed in M2 medium containing $4 \mathrm{mg}$ polyvinylpyrrolidone (PVP) $\mathrm{ml}^{-1}$, collected in sample buffer (Laemmli, 1970), heated to $100^{\circ} \mathrm{C}$ for $3 \mathrm{~min}$ and frozen at $-20^{\circ} \mathrm{C}$. The proteins were separated by electrophoresis in $8 \%(\mathrm{w} / \mathrm{v})$ polyacrylamide (ratio acrylamide:bis-acrylamide was 100:1), containing $0.1 \%(\mathrm{w} / \mathrm{v})$ SDS and electrically transferred to nitrocellulose membranes (Schleicher and Schuell, Düren; pore size $0.45 \mu \mathrm{m})$. After transfer and blocking for $2 \mathrm{~h}$ in $3 \%(\mathrm{w} / \mathrm{v})$ skimmed milk in $10 \mathrm{mmol}$ Tris $\mathrm{I}^{-1}(\mathrm{pH} 7.5)$ and $140 \mathrm{mmol} \mathrm{NaCl} \mathrm{I^{-1 }}$ (TBS) containing $0.1 \%(\mathrm{v} / \mathrm{v})$ Tween-20, the membrane was incubated overnight at $4{ }^{\circ} \mathrm{C}$ with mouse monoclonal anti-BUB1 diluted 1:1000. After three washes of $10 \mathrm{~min}$ each in $0.1 \%(\mathrm{v} / \mathrm{v})$ Tween-20-TBS, the membrane was incubated for $1 \mathrm{~h}$ at room temperature with an antimouse antibody conjugated to horseradish peroxidase (Amersham, Little Chalfont) diluted 1:10 000 in 3\% (w/v) skimmed milk in $0.1 \%(\mathrm{v} / \mathrm{v})$ Tween-20-TBS. The membrane was washed three times in TBS-Tween-20 and processed using either the ECL (Amersham) or the Super Signal (Pierce, Rockford, IL) detection system.

\section{Alkaline phosphatase treatment}

Samples containing 50 oocytes in phosphatase buffer (Boehringer-Mannheim, Mannheim) and 1\% (w/v) SDS (Sigma Chemical Co.) supplemented with anti-proteases were mixed with 1 iu alkaline phosphatase. After $30 \mathrm{~min}$ incubation at $37^{\circ} \mathrm{C}$, the reaction was stopped by adding the same volume of twice concentrated Laemmli buffer. 

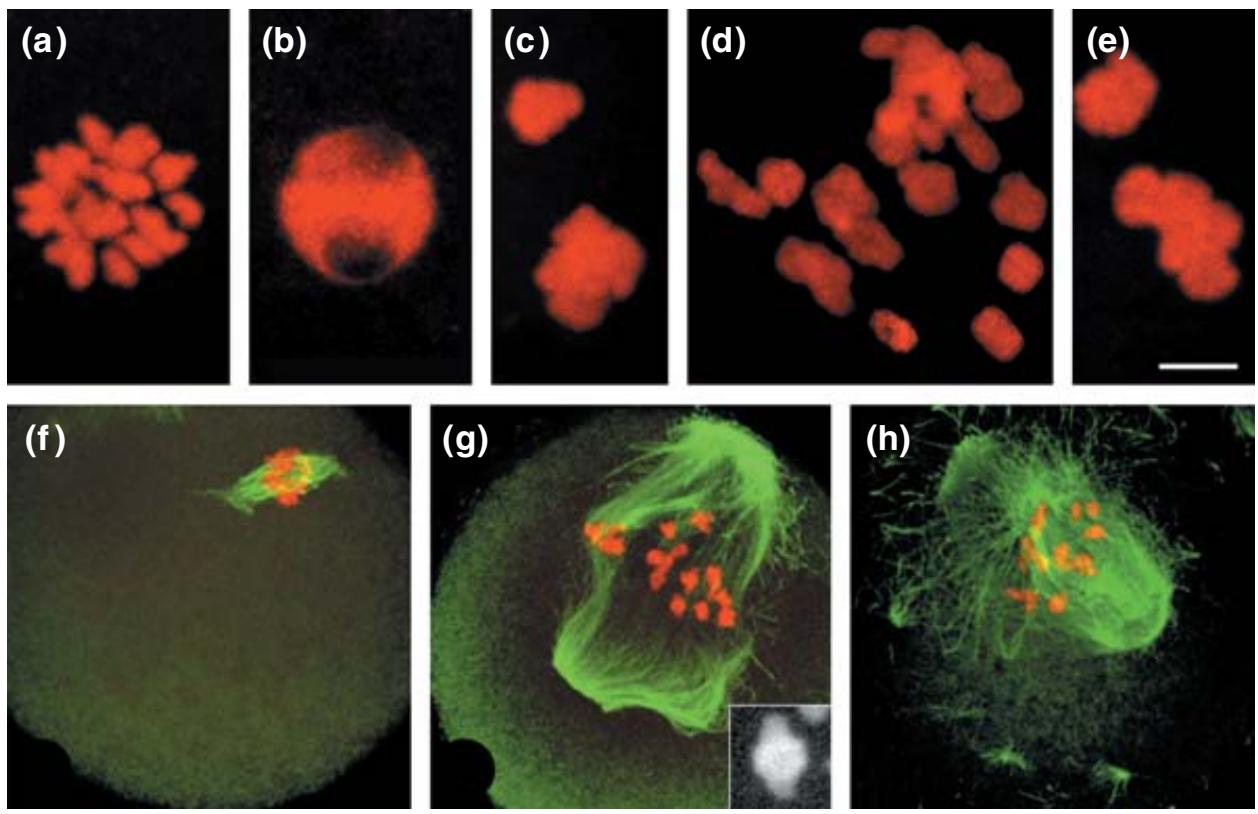

Fig. 1. Functionality of the spindle checkpoint in mouse oocytes at MI. (a) Untreated oocytes: at $11 \mathrm{~h}$ after germinal vesicle breakdown (GVBD), the chromosomes are visible as paired sister chromatids and are aligned on the MII metaphase plate (upper view). (b) Puromycin-treated $\left(20 \mu \mathrm{mol} \mathrm{I} \mathrm{I}^{-1}\right)$ oocytes: at $11 \mathrm{~h}$ after GVBD, the chromatin is decondensed and a pronucleus has formed. (c) Nocodazole-treated $\left(20 \mu \mathrm{mol} \mathrm{I}^{-1}\right)$ oocytes: at $11 \mathrm{~h}$ after GVBD, the chromatin is highly condensed. (d) After brief removal of nocodazole, homologous chromosomes appear to be still clearly paired. (e) Oocytes simultaneously incubated in puromycin $\left(20 \mu \mathrm{mol} \mathrm{I}^{-1}\right)$ and nocodazole $\left(20 \mu \mathrm{mol} \mathrm{I} \mathrm{I}^{-1}\right)$ : the chromatin remains highly condensed at $11 \mathrm{~h}$ after GVBD. (f) Untreated, MII-arrested oocyte. (g) Oocyte treated with paclitaxel $\left(20 \mathrm{nmol} \mathrm{I}^{-1}\right)$ : at $11 \mathrm{~h}$ after GVBD, the chromosomes are located in the area of the metaphase plate. Homologous chromosomes still appear to be clearly paired (inset). (h) Oocytes treated with paclitaxel $\left(20 \mathrm{nmol} \mathrm{I}^{-1}\right)$ and puromycin $\left(20 \mu \mathrm{mol} \mathrm{I}^{-1}\right)$ : at $11 \mathrm{~h}$ after GVBD, the chromosomes are condensed and located in the area of the metaphase plate. $(\mathrm{a}-\mathrm{h})$ Chromatin appears in red and $(\mathrm{f}, \mathrm{g}, \mathrm{h})$ microtubules appear in green. Scale bar represents $5 \mu \mathrm{m}$.

\section{Results}

Functionality of the spindle checkpoint during meiosis I in mouse oocytes

The spindle checkpoint can be activated experimentally by altering spindle microtubule dynamics, and represses both chromosome segregation and MPF inactivation ( $\mathrm{M}$ phase promoting factor; Cleveland et al., 2003). The effect of spindle microtubule depolymerization or stabilization on chromosome segregation and MPF inactivation in maturing mouse oocytes was examined to determine whether this checkpoint is functional during mammalian meiosis.

Oocytes normally extrude their polar body about $8 \mathrm{~h}$ after GVBD. At $11 \mathrm{~h}$ after GVBD, oocytes are arrested in metaphase of the second meiotic $M$ phase (MII), with paired sister chromatids aligned on the metaphase plate (Fig. 1a). In contrast, when oocytes were incubated in nocodazole $\left(20 \mu \mathrm{mol} \mathrm{I}^{-1}\right.$ ) (or $400 \mathrm{nmol} \mathrm{I}^{-1}$, data not shown) from $7 \mathrm{~h}$ to $11 \mathrm{~h}$ after GVBD, the spindle disassembled, polar body extrusion did not occur and the chromatin was highly condensed (Fig. 1c). At $30 \mathrm{~min}$ after nocodazole washout, individual chromosomes were clearly observed as paired homologues (Fig. 1d).

MPF inactivation is only transient between $\mathrm{MI}$ and MII (Verlhac et al., 1994). It can be observed by treating oocytes with inhibitors of protein synthesis at the end of $\mathrm{Ml}$ to induce interphase artificially after $\mathrm{Ml}$ completion. Therefore, to determine whether spindle damage prevents MPF inactivation, oocytes were incubated in the protein synthesis inhibitor puromycin from $7 \mathrm{~h}$ to $11 \mathrm{~h}$ after GVBD, with and without nocodazole $\left(20 \mu \mathrm{mol} \mathrm{I} \mathrm{I}^{-1}\right.$ or $400 \mathrm{nmol} \mathrm{I}^{-1}$ ). In puromycin-treated oocytes, after polar body extrusion, the chromatin decondensed and a pronucleus was observed as an indicator of MPF inactivation (Fig. 1b). In contrast, in oocytes incubated in both puromycin and nocodazole $\left(20 \mu \mathrm{mol} \mathrm{I}^{-1}\right.$ ) (or $400 \mathrm{nmol} \mathrm{I}^{-1}$, data not shown), polar body extrusion was inhibited and the chromatin remained highly condensed in all treated oocytes (Fig. 1e). 
Similar experiments were performed using paclitaxel (a Taxol equivalent) as a microtubule-stabilizing drug. At $11 \mathrm{~h}$ after GVBD, untreated oocytes were arrested in metaphase II (Fig. 1f). In contrast, all oocytes incubated from $6 \mathrm{~h}$ to $11 \mathrm{~h}$ after GVBD in paclitaxel $\left(20 \mathrm{nmol} \mathrm{I}^{-1}\right)$, alone or with puromycin $\left(20 \mu \mathrm{mol} \mathrm{I}^{-1}\right)$, had large spindles with paired homologue chromosomes gathered near the equatorial plane of the spindle (Fig. 1g,h). In conclusion, microtubule depolymerization or stabilization are both able to inhibit chromosome segregation and MPF inactivation, indicating that the spindle checkpoint is functional during the first meiotic $M$ phase in mouse oocytes.

\section{Association of BUB1 with kinetochores during meiotic maturation of oocytes}

The essential spindle checkpoint component BUB1 was also analysed in maturing mouse oocytes. BUB1 localization was investigated using the anti-murine BUB1 monoclonal antibody 4B12 characterized previously in mouse somatic cells (Taylor and McKeon, 1997). During the first meiotic $M$ phase $(\mathrm{MI}), \mathrm{BUB1}$ was observed on the kinetochores from GVBD until metaphase, about $8 \mathrm{~h}$ after GVBD (Fig. 2a-d). During anaphase I, a pool of the protein remained at the kinetochores and disappeared at late anaphase (Fig. 2e,f). During the second meiotic $M$ phase (MII), BUB1 relocalized and remained associated with kinetochores during the metaphase II arrest (Fig. 2g). The protein only disappeared from kinetochores in late anaphase II (Fig. 2i). Treatment of metaphase II-arrested oocytes with nocodazole for $1 \mathrm{~h}$ did not lead to a significant increase in BUB1 content at the kinetochores (Fig. 2h). BUB1 was also observed at both telophase I and II, at the neck of the polar body, at a structure that may correspond to the actin contractile ring but that was not analysed further (Fig. 2f,i). In conclusion, BUB1 associates with kinetochores until anaphase of both meiotic $M$ phases in mouse oocytes.

\section{Phosphorylation of BUB1 during both meiotic $M$ phases}

In somatic cells, experimental spindle checkpoint activation correlates with BUB1 phosphorylation (Taylor and McKeon, 1997). Therefore, BUB1 was analysed by immunoblotting during meiotic maturation. At the GV stage, the protein is already present and its expression remains constant during meiotic maturation (Fig. 3a,b). BUB1 was detected as a single band migrating at $120 \mathrm{kDa}$ at the GV stage. As soon as GVBD occurred, BUB1 shifted to a slower migrating form that was maintained during MI (Fig. 3a) and was also observed during the metaphase II arrest (Fig. 3b). The shift of BUB1 observed during both meiotic $M$ phases was compared with the electrophoretic mobility of the protein after microtubule depolymerization. MI oocytes collected at $7 \mathrm{~h}$ after
GVBD and MII-arrested oocytes were incubated in nocodazole for $3 \mathrm{~h}$. In both cases the electrophoretic mobilities were identical in treated and untreated oocytes (Fig. 3b, lanes 1-4). Finally, the electrophoretic shift was reversed by alkaline phosphatase treatment of metaphase II-arrested oocytes (Fig. 3b, lane 5), demonstrating that this shift corresponded to the phosphorylation of BUB1. In conclusion, during both meiotic $M$ phases, BUB1 is phosphorylated before the onset of anaphase in the same way as upon experimental spindle checkpoint activation.

BUB1 behaviour and the MOS/... MAP kinase pathway

The potential action of the MOS/.../MAP kinase pathway on BUB1 behaviour during meiotic maturation was examined using mos -/- mouse oocytes (Colledge et al., 1994; Hashimoto et al., 1994). In these oocytes, the absence of MAP kinase activation results in failure of metaphase II arrest and in spontaneous activation. After second polar body extrusion, most of the oocytes arrest for at least $10 \mathrm{~h}$ in a third meiotic M phase (MIII), in which they have monopolar spindles (Verlhac et al., 1996).

BUB1 localization was investigated by immunostaining during meiotic maturation of mos $-/-$ and mos +/ oocytes. In mos -/- oocytes, BUB1 associated with kinetochores from GVBD until late anaphase of the first meiotic M phase (Fig. 4a,b), as in mos +/- oocytes (data not shown) or in wild-type oocytes (Fig. 2). In mos -/MIII-arrested oocytes, BUB1 was found on the kinetochores of the sister chromatids (Fig. 4c). BUB1 electrophoretic mobility was compared in mos - /- and wildtype oocytes by immunoblotting during the first meiotic $M$ phase. In both cases, a single band was detected at the GV stage and a shift in electrophoretic mobility was observed after GVBD (Fig. 5). In conclusion, in mos -/oocytes, BUB1 localization and phosphorylation are not impaired compared with mos +/- and wild-type oocytes during meiotic maturation.

\section{Discussion}

The results of the present study indicate that the spindle checkpoint is functional in mouse oocytes. Spindle microtubule depolymerization and stabilization are able to prevent both chromosome segregation and MPF inactivation. Thus, spindle defects are able to prevent onset of anaphase I by triggering a spindle checkpoint arrest. Brunet et al. (1999) observed in MI oocytes that the arrest induced by microtubule depolymerization using nocodazole can be reversed by washing out the drug; in this case, the spindle reforms and anaphase occurs after alignment of the last chromosome on the metaphase plate. Taken together, these data indicate that the spindle checkpoint controls the metaphase I-anaphase I transition in mouse oocytes. 


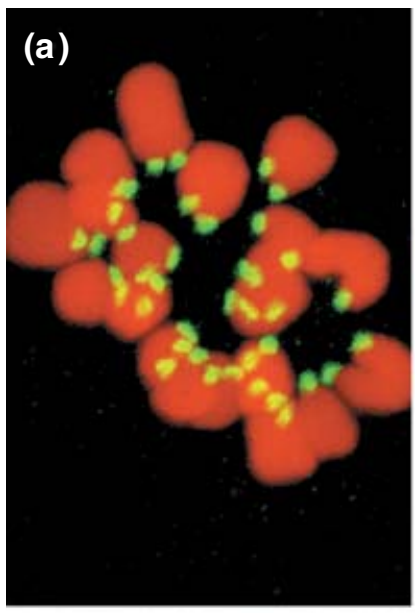

(d)
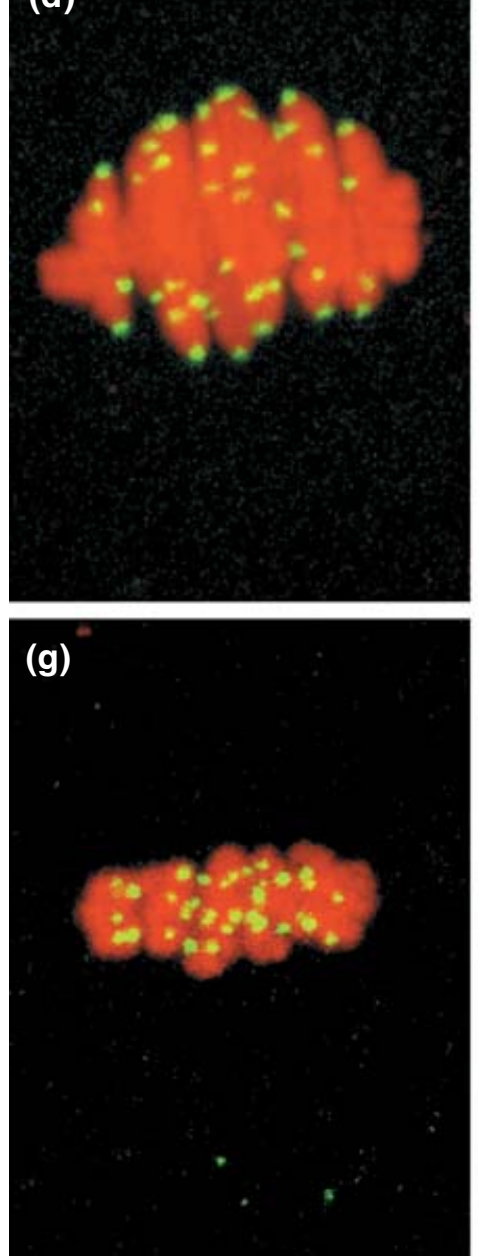

(b)

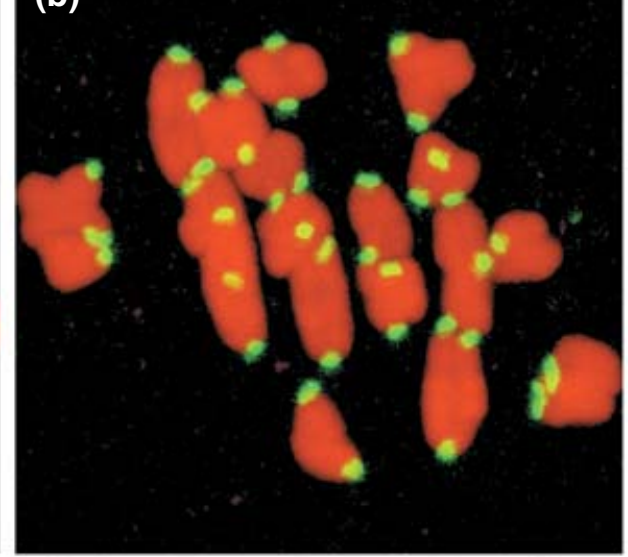

(e)

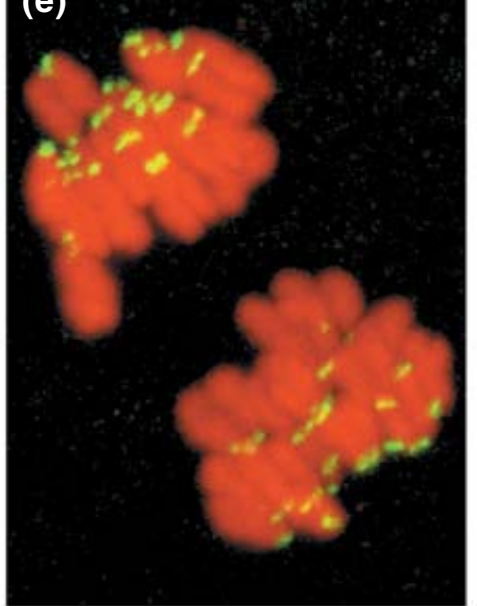

\section{(h)}

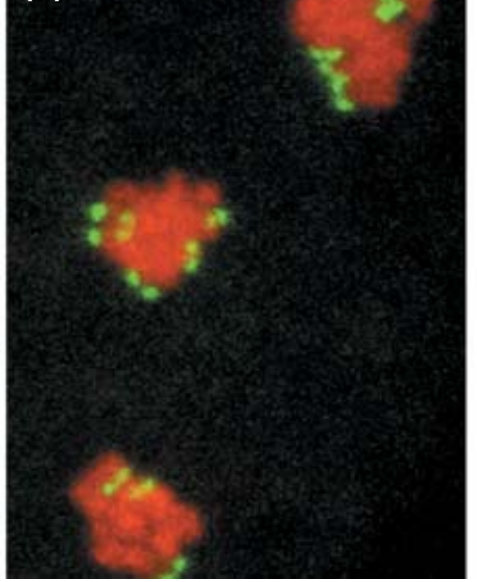

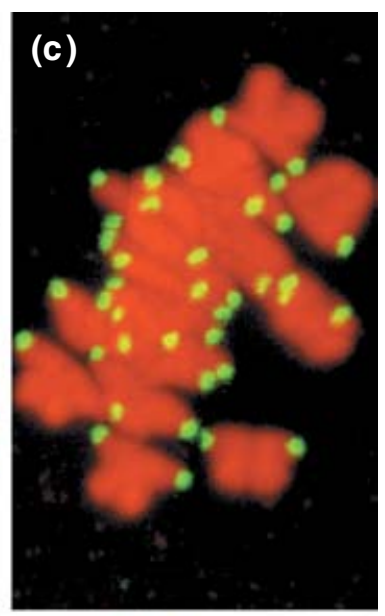

(f)

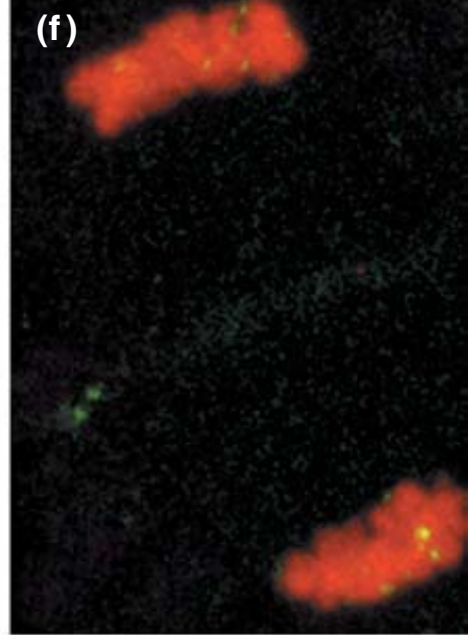

(I)

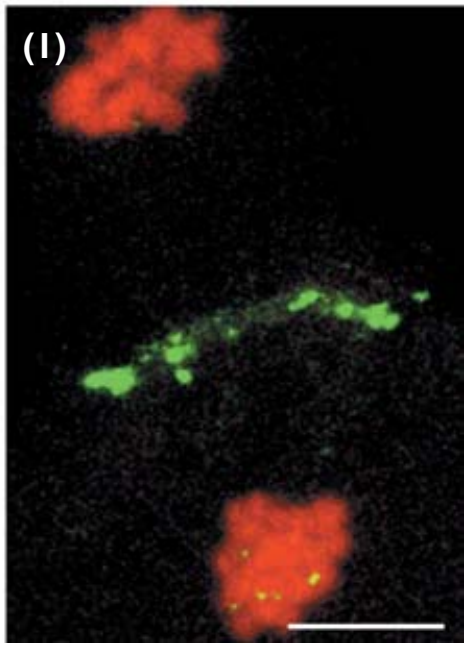

Fig. 2. Association of budding uninhibited by benzimidazole 1 (BUB1) with kinetochores in mouse oocytes. (a) Germinal vesicle breakdown (GVBD). (b) At $3 \mathrm{~h}$ after GVBD, the chromosomes are congressing towards the spindle equator. (c) At $8 \mathrm{~h}$ after GVBD (late prometaphase): before the formation of kinetochore fibres (Brunet et al., 1999), the chromosomes are not yet aligned on the metaphase plate. (d) At $8 \mathrm{~h}$ after GVBD: metaphase. (e) Anaphase. (f) Late anaphase. (g) Metaphase II arrest. (h) Metaphase II-arrested oocytes incubated for $1 \mathrm{~h}$ in nocodazole. (i) Late anaphase II. Red: chromosomes; green: BUB1. Scale bar represents $5 \mu \mathrm{m}$. 
(a)

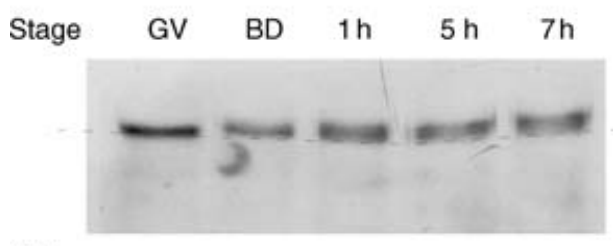

(b)

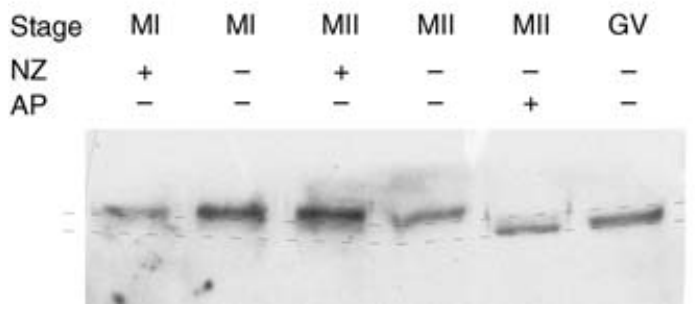

Fig. 3. Transient phosphorylation of budding uninhibited by benzimidazole 1 (BUB1) during meiotic maturation of mouse oocytes. (a) BUB1 immunoblot in MI mouse oocytes. GV: germinal vesicle stage; BD: germinal vesicle breakdown stage. (b) Microtubule depolymerization by nocodazole (NZ) in $\mathrm{MI}$ oocytes, at $7 \mathrm{~h}$ after germinal vesicle breakdown (GVBD; lanes 1 and 2; MI), and MIl-arrested oocytes (lanes 3 and 4), does not modify BUB1 phosphorylation. Alkaline phosphatase (AP) treatment of MII oocytes suppresses the BUB1 electrophoretic shift (lane 5) compared with mobility at the germinal vesicle stage (lane $6 ; \mathrm{GV}$ ). Dashed lines were added to easily visualize the migration shifts. The lines were traced parallel to the protein bands.

The essential spindle checkpoint component BUB1 was characterized in mouse oocytes to support further the existence of the meiotic checkpoint. During both meiotic M phases, BUB1 is associated with kinetochores until anaphase. BUB1 is phosphorylated after microtubule depolymerization, as is also reported in mitosis. Furthermore, BUB1 is phosphorylated to a similar extent during both meiotic $M$ phases before anaphase. In human cells, the phosphorylated form of BUB1 is only readily detectable after spindle damage (Taylor et al., 2001), indicating that it may reflect spindle checkpoint activity. Indeed, BUB1 phosphorylation may be required for the formation of the MAD1-BUB1-BUB3 complex that is crucial for spindle checkpoint function (Brady and Hardwick, 2000). However, BUB1 phosphorylation has also been observed in Xenopus oocytes (Schwab et al., 2001; Sharp-Baker and Chen, 2001), which lack a robust spindle checkpoint (see Minshull et al., 1994). Nevertheless, in this case BUB1 phosphorylation requires the MOS/... MAP kinase pathway (Schwab et al., 2001). In contrast, the results of the present study show, using mos - /- mice, that BUB1 is phosphorylated during the first meiotic $M$ phase independent of the MOS/... MAP kinase pathway. Moreover, after second polar body extrusion, mos -/- oocytes arrest in a third meiotic $M$ phase (MIII) with single chromatids connected to a monopolar spindle for at least $10 \mathrm{~h}$ (Verlhac et al., 1996).
In this case, an abnormal spindle is able to trigger a spindle checkpoint arrest in the absence of the MOS/.../MAP kinase pathway, and thus of CSF. These observations reinforce the contention that BUB1 phosphorylation in $\mathrm{MI}$ mouse oocytes reflects spindle checkpoint activity. In conclusion, our data are strongly indicative that the BUB1-dependent spindle checkpoint is active in mouse oocytes.

It was also observed that a pool of BUB1 persists until late anaphase on the kinetochores. BUB1 in mouse somatic cells appears to leave the kinetochore at metaphase (Taylor and McKeon, 1997). However, a pool of BUB1 is also observed on the kinetochores during anaphase in Saccharomyces pombe (Bernard et al., 1998, 2001) and Caenorhabditis elegans (Basu et al., 1999). These data and our present observations indicate that spindle checkpoint inactivation does not require BUB1 delocalization from the kinetochore.

The existence of a meiotic spindle checkpoint has been documented in various organisms (Bernard et al., 1998; Basu et al., 1999; Yu et al., 1999; Shonn et al., 2000), but its existence in mammalian oocytes remained controversial. In women, an estimated $20 \%$ of pregnancies are aneuploid as result of chromosome segregation errors in oocytes. Most trisomies arise from errors at MI, correlated with increased maternal age (Angell et al., 1994). These observations argue against the functionality of such a checkpoint. Nevertheless, an alternative mechanism for generation of trisomy has been proposed. During the extended dictyate stage in older women, the cohesion within the bivalent complex is severely weakened. Consequently, as oocytes resume meiosis, some bivalents emerge as chromatids unable to hold together as paired homologues. They may act as univalents and be able to silence the spindle checkpoint and divide equationally at anaphase I (Wolstenholme and Angell, 2000).

In addition, in XO mice oocytes, the first meiotic $M$ phase leads to equational division of the univalent $X$ chromosome (30\% of the oocytes), or 'intact segregation' to the egg ( $49 \%$ of the oocytes) or to the polar body (21\% of the oocytes) (LeMaire-Adkins and Hunt, 2000) without any delay in onset of anaphase I. These data may be indicative of the lack of a meiotic spindle checkpoint. Nevertheless, in these oocytes, the $X$ univalent aligns on the Ml metaphase plate (LeMaire-Adkins et al., 1997), most probably with sister kinetochores attached to opposite spindle poles as suggested by further equational segregation in $30 \%$ of the oocytes. It can be proposed that this inappropriate attachment is sufficient to silence the spindle checkpoint and allow onset of anaphase. At anaphase I the cohesion between sister chromatids that is maintained on the centromere (Moore and Orr-Weaver, 1998; Prieto et al., 2001) would counteract the tensions exerted by the kinetochore fibres and prevent proper separation of sister chromatids. In most of the cases, such tensions would be resolved by the loss of connection 

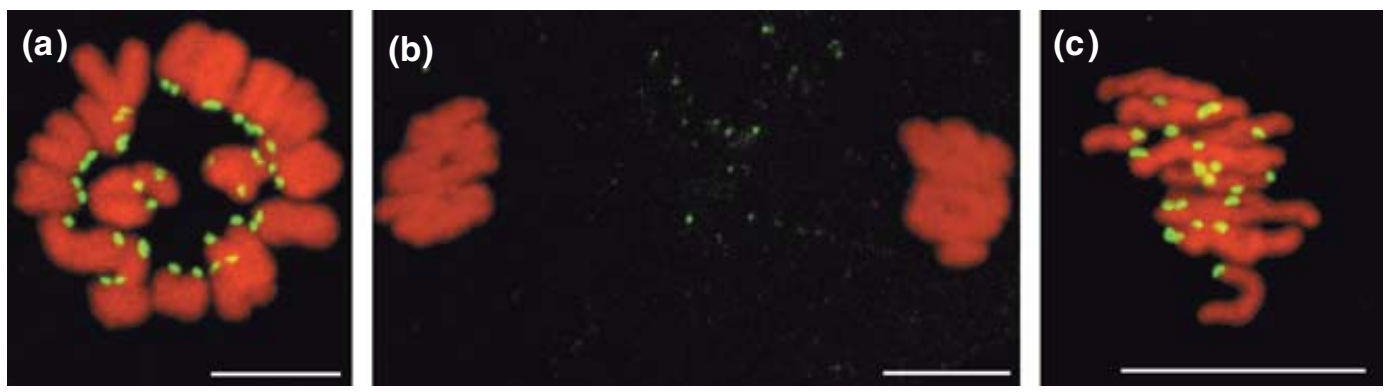

Fig. 4. Localization of budding uninhibited by benzimidazole 1 (BUB1) during meiotic maturation of MOS -/- mouse oocytes. (a) Germinal vesicle breakdown. (b) Late anaphase I. (c) MIII-arrested oocyte. Red: chromosomes; green: BUB1. Scale bars represent $5 \mu \mathrm{m}$.

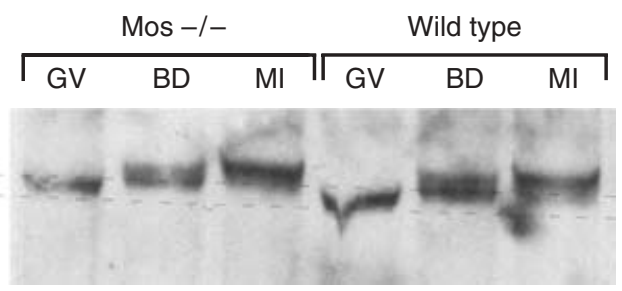

Fig. 5. Phosphorylation of budding uninhibited by benzimidazole 1 (BUB1) in MOS -/- and wild-type mouse oocytes during MI. BUB1 immunoblot in MOS -/- and wild-type mouse oocytes. In MOS -/- mouse oocytes, the migrating forms of BUB1 detected at the germinal vesicle (GV; lane 1), germinal vesicle breakdown (BD; lane 2$)$ and GVBD + $7 \mathrm{~h}(\mathrm{Ml}$; lane 3$)$ stages are identical to those detected in wild-type oocytes at the corresponding stages (lanes 4-6). Dashed lines were added to visualize the migration shifts. The lines were traced parallel to the protein bands.

between one kinetochore and its spindle pole, and would lead to 'intact segregation' of the $X$ univalent.

In conclusion, the existence of an active spindle checkpoint in mammalian oocytes that may depend on BUB1 indicates that the chromosome segregation errors observed in mammalian oocytes probably occur during anaphase I. The errors most probably result from the weakening of the cohesion within the bivalent correlated with maternal age. The bivalents correctly connected to the spindle poles as univalents may silence the spindle checkpoint and then divide equationally. The mechanism of such a process remains to be investigated.

As in most vertebrates, mouse oocytes are arrested at metaphase of the second meiotic $M$ phase until fertilization or parthenogenetic activation. During this arrest, a high MPF activity is maintained by CSF (cytostatic factor), a factor which involves the MOS/... /MAP kinase cascade (Sagata, 1998). BUB1 is present on the kinetochores of MII-arrested oocytes and only leaves the kinetochores at late anaphase, as during $\mathrm{MI}$ and as observed in other organisms (see above). BUB1 remains phosphorylated during the MII arrest as it is upon microtubule depolymerization. An intact spindle is required for egg activation (Winston et al., 1995), indicating that the spindle checkpoint can be activated during the MII arrest. However, the perfect alignment of the chromosomes observed by videomicroscopy during this arrest (Brunet et al., 1999) indicates that the spindle checkpoint has probably been turned off. BUB1 phosphorylation is required for spindle checkpoint activity (Yamaguchi et al., 2003). However, it is not known whether its dephosphorylation is necessary to turn off the checkpoint or whether it takes place at anaphase when BUB1 leaves the kinetochore. Finally, cyclin B degradation has been shown to occur during the MII arrest in mouse oocytes, and MPF activity is maintained through an equilibrium between cyclin B degradation and synthesis (Kubiak et al., 1993). In all systems analysed so far, spindle checkpoint activity completely prevents cyclin B degradation. These data provide strong evidence against involvement of a simple spindle checkpoint in CSF arrest.

Moreover, an alternative pathway for BUB1 phosphorylation, dependent on the MOS/.../MAP kinase pathway, has been described in Xenopus oocytes (Schwab et al., 2001). In addition, BUB1 appears to be required to establish the MII arrest in Xenopus egg extract (Tunquist et al., 2002). In mouse mos -/- oocytes, BUB1 phosphorylation occurs in $\mathrm{MI}$ and during the metaphase III arrest, when the spindle checkpoint is active. However, the lack of MII arrest in these oocytes did not allow us to investigate BUB1 phosphorylation biochemically at that stage. In conclusion, our data indicate that BUB1 may contribute to metaphase arrest in mouse oocytes independent of checkpoint activation. The putative contribution of BUB1 in this arrest and a possible regulation by the MOS/... /MAP kinase pathway remains to be investigated through gain and loss of function experiments.

The authors would like to thank J. Kubiak for fruitful discussions during the course of experiments. S. Brunet and G. Pahlavan are fellows from 'la Ligue Nationale contre le Cancer' and the 'Fondation pour la Recherche Médicale', respectively. This work was supported by a grant from the 'Association pour la Recherche contre le Cancer' to B. Maro. 


\section{References}

Abruzzo MA and Hassold TJ (1995) Etiology of nondisjunction in humans Environmental Molecular Mutagens 25 38-47

Amon A (1999) The spindle checkpoint Current Opinion in Genetics and Development 9 69-75

Angell RR, Xian J, Keith J, Ledger W and Baird DT (1994) First meiotic division abnormalities in human oocytes: mechanism of trisomy formation Cytogenetics and Cell Genetics 65 194-202

Basu J, Bousbaa H, Logarinho E, Li Z, Williams BC, Lopes C, Sunkel CE and Goldberg ML (1999) Mutations in the essential spindle checkpoint gene bub1 cause chromosome missegregation and fail to block apoptosis in Drosophila. Journal of Cell Biology 146 13-28

Bernard P, Hardwick K and Javerzat JP (1998) Fission yeast bub1 is a mitotic centromere protein essential for the spindle checkpoint and the preservation of correct ploidy through mitosis Journal of Cell Biology 143 1775-1787

Bernard P, Maure JF and Javerzat JP (2001) Fission yeast Bub1 is essential in setting up the meiotic pattern of chromosome segregation Nature Cell Biology 3 522-526

Brady DM and Hardwick KG (2000) Complex formation between Mad1p, Bub1p and Bub3p is crucial for spindle checkpoint function Current Biology 10 675-678

Brunet S, Maria AS, Guillaud P, Dujardin D, Kubiak JZ and Maro B (1999) Kinetochore fibers are not involved in the formation of the first meiotic spindle in mouse oocytes, but control the exit from the first meiotic $M$ phase Journal of Cell Biology 146 1-12

Cleveland DW, Mao Y and Sullivan KF (2003) Centromeres and kinetochores: from epigenetics to mitotic checkpoint signaling Cell 112 407-421

Colledge WH, Carlton MB, Udy GB and Evans MJ (1994) Disruption of c-mos causes parthenogenetic development of unfertilized mouse eggs Nature 370 65-68

Cuthbertson KS (1983) Parthenogenetic activation of mouse oocytes in vitro with ethanol and benzyl alcohol Journal of Experimental Zoology 226 311-314

Eichenlaub-Ritter U, Chandley AC and Gosden RG (1988) The CBA mouse as a model for age-related aneuploidy in man: studies of oocyte maturation, spindle formation and chromosome alignment during meiosis Chromosoma 96 220-226

Hashimoto N, Watanabe N, Furuta Y et al. (1994) Parthenogenetic activation of oocytes in c-mos-deficient mice Nature $\mathbf{3 7 0}$ 68-71

Hoyt MA, Totis L and Roberts BT (1991) S. cerevisiae genes required for cell cycle arrest in response to loss of microtubule function Cell $\mathbf{6 6}$ 507-517

Hunt PA and LeMaire-Adkins R (1998) Genetic control of mammalian female meiosis Current Topics in Developmental Biology 37359 381

Kubiak JZ, Weber M, de Pennart H, Winston NJ and Maro B (1993) The metaphase II arrest in mouse oocytes is controlled through microtubuledependent destruction of cyclin B in the presence of CSF EMBO Journal 12 3773-3778

Laemmli UK (1970) Cleavage of structural proteins during the assembly of the head of bacteriophage T4 Nature 227 680-685

LeMaire-Adkins R and Hunt PA (2000) Nonrandom segregation of the mouse univalent $\mathrm{X}$ chromosome: evidence of spindle-mediated meiotic drive Genetics 156 775-783

LeMaire-Adkins R, Radke K and Hunt PA (1997) Lack of checkpoint control at the metaphase/anaphase transition: a mechanism of meiotic nondisjunction in mammalian females Journal of Cell Biology 1391611 1619

Li R and Murray AW (1991) Feedback control of mitosis in budding yeast Cell 66 519-531 [published erratum appears in Cell 1994 Oct 21; 79(2):following 388].

Maro B, Johnson MH, Pickering SJ and Flach G (1984) Changes in actin distribution during fertilization of the mouse egg Journal of Embryology and Experimental Morphology 81 211-237
Minshull J, Sun H, Tonks NK and Murray AW (1994) A MAP kinasedependent spindle assembly checkpoint in Xenopus egg extracts Cell 79 475-486

Moore DP and Orr-Weaver TL (1998) Chromosome segregation during meiosis: building an unambivalent bivalent Current Topics in Developmental Biology 37 263-299

Prieto I, Suja JA, Pezzi N, Kremer L, Martinez AC, Rufas JS and Barbero JL (2001) Mammalian STAG3 is a cohesin specific to sister chromatid arms in meiosis I Nature Cell Biology 3 761-766

Sagata N (1998) Introduction: meiotic maturation and arrest in animal oocytes Seminars in Cellular Developmental Biology 9 535-537

Schwab MS, Roberts BT, Gross SD, Tunquist BJ, Taieb FE, Lewellyn AL and Maller JL (2001) Bub1 is activated by the protein kinase p90(Rsk) during Xenopus oocyte maturation Current Biology 11 141-150

Sharp-Baker H and Chen RH (2001) Spindle checkpoint protein Bub1 is required for kinetochore localization of Mad1, Mad2, Bub3, and CENP$\mathrm{E}$, independently of its kinase activity Journal of Cell Biology 1531239 1250

Shonn MA, McCarroll R and Murray AW (2000) Requirement of the spindle checkpoint for proper chromosome segregation in budding yeast meiosis Science 289 300-303

Skoufias DA, Andreassen PR, Lacroix FB, Wilson L and Margolis RL (2001) Mammalian mad2 and bub1/bubR1 recognize distinct spindleattachment and kinetochore-tension checkpoints Proceedings National Academy of Sciences USA 98 4492-4497

Taylor SS and McKeon F (1997) Kinetochore localization of murine Bub1 is required for normal mitotic timing and checkpoint response to spindle damage Cell 89 727-735

Taylor SS, Hussein D, Wang Y, Elderkin S and Morrow CJ (2001) Kinetochore localisation and phosphorylation of the mitotic checkpoint components Bub1 and BubR1 are differentially regulated by spindle events in human cells Journal of Cell Science 114 4385-4395

Tunquist BJ, Schwab MS, Chen LG and Maller JL (2002) The spindle checkpoint kinase bub1 and cyclin e/cdk2 both contribute to the establishment of meiotic metaphase arrest by cytostatic factor Current Biology 12 1027-1033

Verlhac MH, Kubiak JZ, Clarke HJ and Maro B (1994) Microtubule and chromatin behavior follow MAP kinase activity but not MPF activity during meiosis in mouse oocytes Development 120 1017-1025

Verlhac MH, Kubiak JZ, Weber M, Geraud G, Colledge WH, Evans M] and Maro B (1996) Mos is required for MAP kinase activation and is involved in microtubule organization during meiotic maturation in the mouse Development 122 815-822

Waters JC, Chen RH, Murray AW, Gorbsky GJ, Salmon ED and Nicklas RB (1999) Mad2 binding by phosphorylated kinetochores links error detection and checkpoint action in mitosis Current Biology 9 649652

Whittingham DG (1971) Culture of mouse ova Journal of Reproduction and Fertility Supplement 14 7-21

Winston NJ, McGuinness O, Johnson MH and Maro B (1995) The exit of mouse oocytes from meiotic $M$-phase requires an intact spindle during intracellular calcium release Journal of Cell Science 108 143-151

Wolstenholme J and Angell RR (2000) Maternal age and trisomy - a unifying mechanism of formation Chromosoma 109 435-438

Yamaguchi S, Decottignies A and Nurse P (2003) Function of Cdc2pdependent Bub1p phosphorylation and Bub1p kinase activity in the mitotic and meiotic spindle checkpoint EMBO Journal 22 1075-1087

Yu HG, Muszynski MG and Kelly Dawe R (1999) The maize homologue of the cell cycle checkpoint protein MAD2 reveals kinetochore substructure and contrasting mitotic and meiotic localization patterns Journal of Cell Biology 145 425-435

Received 1 May 2003.

First decision 11 June 2003.

Revised manuscript received 25 June 2003.

Accepted 2 July 2003. 